\title{
Tailoring dual reversal modes by helicity control in ferromagnetic nanotubes
}

\author{
H. D. Salinas $\odot,{ }^{1, *}$ J. Restrepo,,$^{1, \dagger}$ and Òscar Iglesias ${ }^{2, \ddagger}$ \\ ${ }^{1}$ Grupo de Magnetismo y Simulación G+, Instituto de Física, Universidad de Antioquia, A.A. 1226, Medellín, Colombia \\ ${ }^{2}$ Departament de Física de la Matèria Condensada and Institut de Nanociència i Nanotecnologia, \\ Universitat de Barcelona, Av. Diagonal 647, 08028 Barcelona, Spain
}

(Received 10 April 2019; revised manuscript received 27 January 2020; accepted 28 January 2020; published 13 February 2020)

\begin{abstract}
We investigate the effects of the competition between exchange $(J)$ and dipolar $(D)$ interactions on the magnetization reversal mechanisms of ferromagnetic nanotubes. Using first atomistic Monte Carlo simulations for a model with Heisenberg spins on a cylindrical surface, we compute hysteresis loops for a wide range of the $\gamma=D / J$ parameter, characterizing the reversal behavior in terms of the cylindrical magnetization components along the tube length. For $\gamma$ 's close to the value for which helical $(H)$ states are energetically favorable at zero applied field, we show that the hysteresis loops can occur in four different classes that are combinations of two reversal modes with well-differentiated coercivities with probabilities that depend on the tube length and radius. This variety in the reversal modes is found to be linked to the metastability of the $H$ states during the reversal that induces different paths followed along the energy landscape as the field is changed. We further demonstrate that reversal by either of the two modes can be induced by tailoring the nanotube initial state so circular states with equal or contrary chirality are formed at the ends, thus achieving low or high coercive fields at will without changing $\gamma$. Finally, the results of additional micromagnetic simulations performed on tubes with a similar aspect ratio show that dual switching modes and its tailoring can also be observed in tubes of microscopic dimensions.
\end{abstract}

DOI: 10.1103/PhysRevB.101.054419

\section{INTRODUCTION}

Magnetic nanotubes have gained increasing interest in the last years from fundamental and technological standpoints due to their double potential functionality arising from their characteristic inner and outer surfaces [1,2]. Recent advances in the experimental methods for fabrication of magnetic tubes and decoration techniques currently allow one to synthesize tubular and cylindric nanostructures by means of different routes [3-8] with a high degree of control on their composition and geometry. The core-free aspect of the tubes as compared to their filled counterparts or nanowires allows, in principle, a fast reversal process with a coercivity that can be controlled by changing the shape factor or the aspect ratio. The variety of magnetic states that can be achieved in these structures have been exploited in sensors, logical devices [9], high-density magnetic memories [10], and even for magnetic hyperthermia and drug release [11-13].

In such systems, vortex $(V)$ and helical $(H)$ states have been predicted to occur theoretically by different methods [14-23]. In particular, vortex states are relevant for magnetic storage purposes since, being flux-closure configurations, they do not produce stray fields, avoiding the consequent leakage of magnetic flux spreading outward from the tube. Advances in imaging and characterization techniques have made pos-

\footnotetext{
*hernan.salinas@udea.edu.co

†johans.restrepo@udea.edu.co

‡oscar@ffn.ub.es; http://www.ffn.ub.es/oscar; http://nanomagn.blogspot.com
}

sible the direct visualization of magnetic configurations in individual nanotubes, confirming the formation of the abovementioned $V$ and $H$ states at different stages of the reversal process [24-29]. Several studies have reported field-driven magnetic-switching mechanisms of individual nanotubes having typical lengths in the range from $0.5 \mu \mathrm{m}$ to some tens of $\mu \mathrm{m}$, radii from $100-500 \mathrm{~nm}[30,31]$, and thicknesses from $10-70 \mathrm{~nm}[27,28,32,33]$, with enough resolution to distinguish the nucleation of different states during reversal and the propagation of domain walls. Recent studies have shown that it is even possible to register hysteresis loops of a single-molecule magnet (SMM) [34].

Given the length scales of real tubes, theoretical understanding of this subject has been gained mainly by using micromagnetic simulations [35]. Apart from these, analytical calculations based on continuum approximations [16,17,36-41] have also succeeded in describing the main phenomenology. Other theoretical methods $[42,43]$ or simulation techniques [44-47] have also partially addressed these issues. However, most of the mentioned works focused on the influence of geometric parameters of the tubes or wires on the reversal mechanisms for a given material and the possibility to explore the range of material parameters for which certain reversal modes can appear has not been exploited so far.

Therefore, the objective of the present paper is to study how the competition between the two main competing energies (given by the parameter $\gamma=D / J$, the ratio of dipolar $(D)$ to exchange $(J)$ interactions between spins) may be used to tune or induce certain reversal modes when a magnetic field is applied along the nanotube axis. Differentiating from most of the above-mentioned works, we use both a 
micromagnetic approach as well as an atomistic Monte Carlo (MC) lattice model, performing simulations of hysteresis loops for certain representative geometric parameters of the tubes and considering values of $\gamma$ within a range that can be relevant to real materials. An atomistic approach allows us to tackle finite-size effects, besides the accessibility to systems at a nanoscopic level of a few nanometers where discreteness becomes relevant, different from the micromagnetic approach where solution of the equations demands the consideration of a continuum model. Additionally, the consideration of a range of $\gamma$ values allows us to mimic a family of tubes with either different values of $J$ or $\mu$ or the nearest-neighbor distance, which can in principle be tailor-made. The effect of geometrical parameters like the aspect ratio is also considered. We discover that for a certain range of $\gamma$ 's, dual reversal modes of low and high coercivity appear as a consequence of the metastability of helical and vortex spin configurations formed during the first stages of the magnetization switching. The same phenomenology is also investigated for FeCo tubes at a micromagnetic level. In both cases, the chirality of helical and vortex spin configurations is a key factor to understand the two reversal mechanisms.

The rest of the paper is organized as follows: In Sec. II, we present the model and physical insights involved in the MC and micromagnetic simulations. In Sec. III, methodology and computational details are presented. In Sec. IV, we start by analyzing the results of the MC simulated hysteresis loops, we show a procedure to tailor the different reversal modes, and end up presenting micromagnetic simulation results that demonstrate that a similar phenomenology can show up in $\mathrm{FeCo}$ tubes with sizes of tens of nanometeres. We finish with a discussion about the relevance of these results as a mechanism for coercivity enhancement and we present the main conclusions.

\section{MODEL}

\section{A. Monte Carlo simulation}

As it concerns the MC simulation approach, a singlespin flip Metropolis dynamics and a three-dimensional (3D) classical Heisenberg Hamiltonian were implemented. The Hamiltonian reads as follows:

$$
\mathcal{H}=E_{\text {ex }}+E_{\text {dip }}+E_{Z},
$$

where $E_{\mathrm{ex}}$ stands for short-range exchange coupling between 3D classical Heisenberg nearest-neighbors spins $\left(S_{x}, S_{y}, S_{z}\right)$,

$$
E_{\mathrm{ex}}=-\sum_{\langle i, j\rangle} J_{i j} \vec{S}_{i} \cdot \vec{S}_{j},
$$

being $J_{i j}=J>0$ the ferromagnetic (FM) exchange constant. This constant was fixed at $10 \mathrm{meV}$, a typical value of FM systems.

The second term $E_{\text {dip }}$ is the long-range magnetic dipolar interaction given by

$$
E_{\mathrm{dip}}=D \sum_{i<j}\left(\frac{\vec{S}_{i} \cdot \vec{S}_{j}-3\left(\vec{S}_{i} \cdot \hat{r}_{i j}\right)\left(\vec{S}_{j} \cdot \hat{r}_{i j}\right)}{\left|\vec{r}_{i j}\right|^{3}}\right),
$$

where $\vec{r}_{i j}$ is the relative vector between $i$ and $j$ positions and summation is over all the sets of pairs $(i, j)$, taking care to count pair interactions once. Making explicit the fact that the pair distance vector can be considered as a multiple of a minimum distance $a$ between nearest neighbors, which can eventually coincide with a lattice parameter, depending on the type of system, we can define an atomistic dipolar coupling strength parameter in energy units as

$$
D=\frac{\mu_{0} \mu^{2}}{4 \pi a^{3}},
$$

with $\mu_{0}$ and $\mu$ being the vacuum permeability and the magnetic moment per spin, respectively. Thus, we can define the dimensionless parameter $\gamma=D / J$ to quantify the degree of competition between long-range and short-range interactions. In this paper, $\gamma$ was set to vary between 0.01 and 0.07 , for which circular states $(V$ and $H)$ can appear [23] and therefore it is expected that competition plays a relevant role on the magnetization reversal mechanisms.

Finally, $E_{Z}$ is the Zeeman interaction of the spins with a uniform external field $\vec{H}$ applied along the main axis of the tube, given by

$$
E_{Z}=-\sum_{i} \mu \vec{S}_{i} \cdot \vec{H}
$$

\section{B. Micromagnetics}

This approach was employed to simulate tubes of some tens of nanometers, i.e., one order of magnitude greater than those simulated atomistically via MC. Both zero temperature and finite-temperature micromagnetic simulations were performed. For the latter, a thermal solver to the Landau-Lifshitz-Gilbert (LLG) equation was employed. Magnetostatic, exchange, and Zeeman energies were considered. The LLG equation, accounting for the magnetization dynamics of the system, was solved by using both a finite-difference method based on the object-orientedmicromagnetic-framework (OOMMF) [48],

$$
\frac{\mathrm{d} \vec{M}}{\mathrm{~d} t}=-\left|\gamma_{L L}\right| \vec{M} \times \vec{H}_{\mathrm{eff}}-\frac{\left|\gamma_{L L}\right| \alpha}{M} \vec{M} \times\left(\vec{M} \times \vec{H}_{\mathrm{eff}}\right),
$$

where $\vec{M}$ is the magnetic moment per cell, $\gamma_{L L}$ and $\alpha$ are the gyromagnetic ratio and the Gilbert damping parameter, respectively, whereas $\vec{H}_{\text {eff }}$ is an effective field that represents all the generalized forces acting on every magnetic moment. Temperature is included in the effective field through a fluctuating or stochastic term $h_{\text {fluc }}$ for which a Boltzmann distribution is assumed for the spin ensemble and the stochastic field is modeled as white noise. The variance of the fluctuating field is given by

$$
\sigma_{h_{\text {fuc }}}^{2}=\frac{\alpha}{1+\alpha^{2}} \frac{2 k_{B} T}{\gamma_{L L} M_{s} V} .
$$

In particular, we examined hysteresis loops and the time evolution of the magnetization at a temperature $T=4 \mathrm{~K}$. Loops were repeated for different values of the random number generator used to generate the stochastic thermal fields. We obtained also equilibrium magnetic configurations using the zero-temperature minimization driver. 


\section{METHODOLOGY AND COMPUTATIONAL DETAILS}

\section{A. Monte Carlo simulation}

Single-wall nanotubes were modeled as shown in a previous work [14] by rolling a square lattice along the $(1,1)$ direction to get a zigzag-terminated 3D tube with symmetry axis along the $z$ direction. We demonstrated in Ref. [23] that such spatial distribution of spins possesses a lower dipolar energy than that of a columnar stacked realization.

Tube dimensions are determined by the pairs $\left(N, N_{z}\right), N$ being the number of spins per layer and $N_{z}$ the number of layers accounting for the height or length of the tubes. In particular, tubes having dimensions $(8,20),(8,15),(8,14)$, and $(8,8)$ were considered, with aspect ratios given by the quotient $N_{z} / N$. Length and radius are given, respectively, by $l=\left(N_{z}-1\right) \sqrt{2} a / 2$ and $R=\sqrt{2} N a /(2 \pi)$ [23].

We have conducted standard Metropolis MC simulations to obtain the hysteresis loops at a fixed low temperature $T=$ $0.1 J / k_{B}$ by cycling the magnetic field in constant steps $\delta H$ between 0.02 and 0.1 , depending on the field region, while performing thermodynamic averages of several components of the magnetization and its rotational. Since thermodynamic states in the hysteresis loops are not strictly equilibrium states, up to 100 different runs per loop were performed to carry out configurational averages with the corresponding error bars calculations. The maximum number of MC steps $\left(5 \times 10^{3}\right)$ and those discarded for thermalization $\left(3 \times 10^{3}\right)$ were kept fixed for all the hysteresis loops.

As already proposed in our previous work [23], to analyze the texture of noncollinear spin configurations during reversal modes, more concretely $V$ or $H$ states, we use also the vorticity order parameter defined as a discretized version of the curl of the magnetization $\vec{\rho}=\vec{\nabla} \times \vec{M}$, that quantifies the vorticity, helicity, or degree of circularity of the magnetic moments (similar to a toroidal moment [49]). Chirality can be characterized in terms of the sign of the azimuthal component of the magnetization $m_{\phi}$. Intermediate configurations attained during the reversal process of the hysteresis loops are tracked by expressing magnetic moments in usual cylindrical coordinates [23] that are better suited to describe $V$ and $H$ states. Thus, at every spin site $\vec{r}_{i}=$ $\left(R \cos \varphi_{i}, R \sin \varphi_{i}, z_{i}\right)$, where $R$ is the tube radius and $\varphi_{i}$ is the respective azimuthal space coordinate, a local reference frame was considered so the spin vector components read $\vec{S}_{i}=$ $\left(S_{z_{i}}, S_{\phi_{i}}, S_{\rho_{i}}\right)=\left(\cos \theta_{i}, \sin \theta_{i} \sin \phi_{i}, \sin \theta_{i} \cos \phi_{i}\right)$ (see Fig. 1 for angle definitions). Therefore, a full characterization of spin configurations during the switching modes can be obtained both by plotting the average polar angle $\langle\theta\rangle$ and the $m_{\phi}$ component per layer for each $z$ value along the entire length of the tube.

\section{B. Micromagnetic simulations}

To investigate both the hysteretic behavior of tubes with greater dimensions and their associated reversal modes, we consider FeCo tubes having an internal diameter of $15 \mathrm{~nm}$, an external one of $21 \mathrm{~nm}$, and a height of $52.5 \mathrm{~nm}$, with an aspect ratio very close to that of a $(8,20)$ tube were considered. Simulations were performed considering magnetostatic, exchange, and Zeeman energies with material parameters close

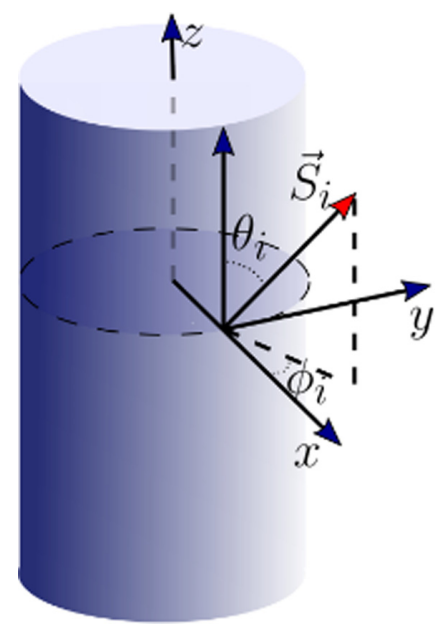

FIG. 1. Local reference frame at a spin site where the magnetic moment is given by $\vec{S}_{i}$, showing the coordinates to describe magnetic configurations, with $\theta$ and $\phi$ the polar and azimuthal spin angles, respectively.

to that of FeCo, i.e., stiffness constant $A=1.08 \times 10^{-11} \mathrm{~J} / \mathrm{m}$, saturation magnetization $M_{s}=1.83 \times 10^{6} \mathrm{~A} / \mathrm{m}$. The smallest mesh size was set at $1.5 \mathrm{~nm}$, below the exchange length of the material.

\section{RESULTS}

\section{A. Hysteresis loops from MC simulations}

In our recent work [23], we established phase diagrams for the zero-field equilibrium configurations for nanotubes with competing exchange and dipolar interactions depending on the value of $\gamma$ and geometric characteristics of the tubes. In particular, independently of the tube length and radius, at low $\gamma$, we found FM ground states along the tube axis while, for large enough $\gamma, V$ states were found to become stable. Interestingly, for a range of $\gamma$ around a critical value $\gamma^{\star}$ that depends on the geometric parameters of the tubes, states $H$ with helical order appear. Therefore, since it is possible to stabilize circular states in tubular structures from which a switching mode can be initiated, it is expected that the magnetization reversal mechanisms of the nanotubes under the application of a magnetic field depend on the $\gamma$ value. To study this, we first simulate low-temperature $\left(T=0.1 J / k_{B}\right)$ hysteresis loops for several values of $\gamma$, taking as a reference a $(8,15)$ tube, for which we found $\gamma^{\star} \simeq 0.035$ [23]. The results of calculated hysteresis loops averaged over 30 runs, in which the initial random-number-generator seed was changed, are shown in Fig. 2 for some selected values of $\gamma$.

For low $\gamma$ values, when exchange interaction is dominant, the tubes behave as collinear ferromagnets reversing their magnetization coherently. Hysteresis loops exhibit a high degree of squareness and appreciable but low values of the vorticity only around the coercive fields. On increasing $\gamma$, the coercive field diminishes as a consequence of the spin canting induced by dipolar interaction at the tube ends, where nucleation begins, which facilitates the spin reversal. Even though the shape of the hysteresis loops in the range $\gamma \leqslant 0.03$ is qualitatively similar, suggesting that the reversal mechanisms 

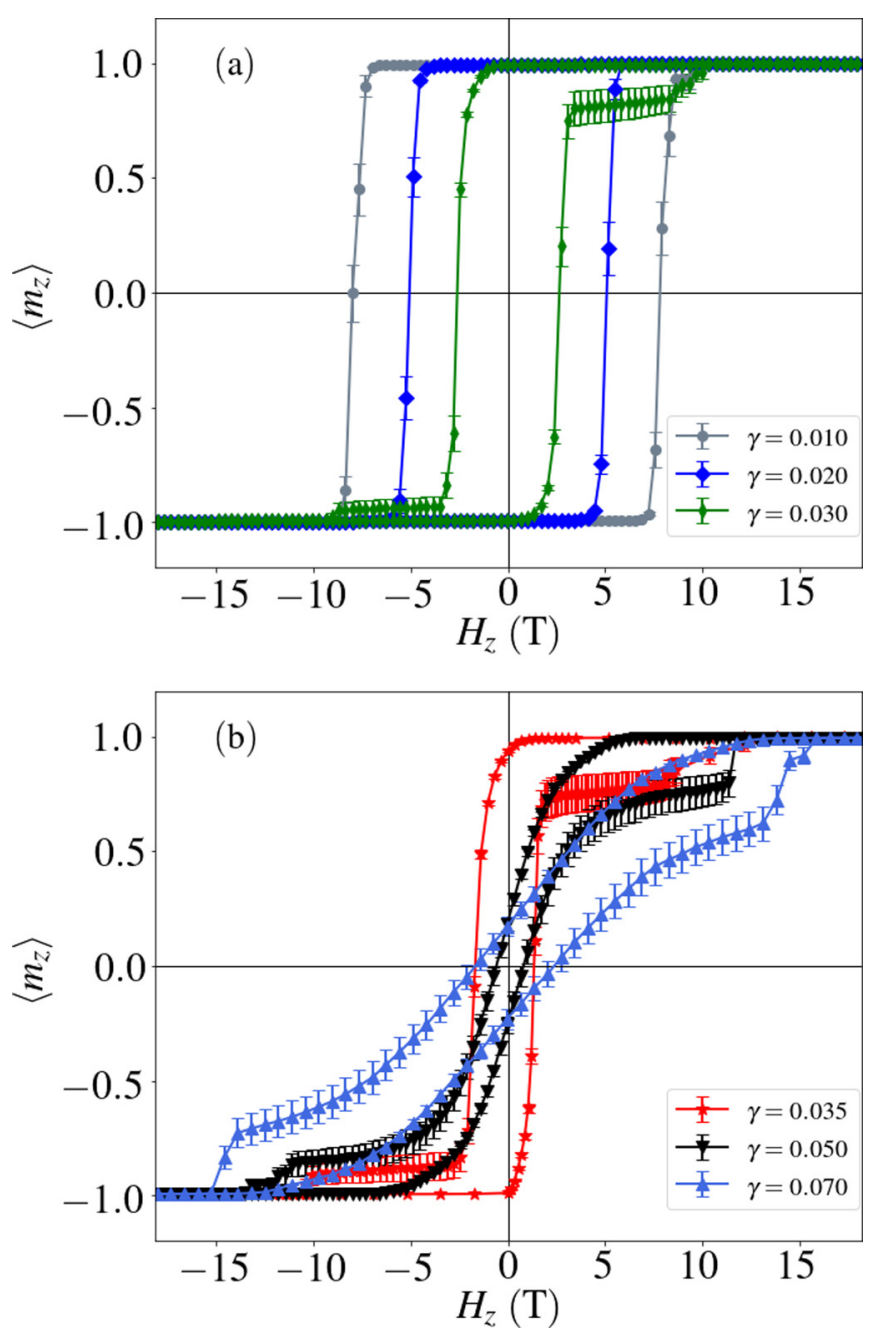

FIG. 2. Low-temperature hysteresis loops averaged over 30 different runs for six different values of $\gamma$ for a $(8,15)$ tube with the field applied parallel along the tube axis.

are preserved, microscopically some differences progressively emerge as indicated by the increasing height of the $\langle|\vec{\rho}|\rangle$ peaks near the coercive fields in Fig. 3(a). This indicates the appearance of intermediate circular magnetic states during the switching process, close to the coercive field. For values of $\gamma \approx \gamma^{\star}=0.035$ for the $(8,15)$ tube, the averaged loops become asymmetric and regions with considerable error bars appear progressively, pointing to variations in the inversion modes from run to run. Bigger error bars are linked to a higher metastability of the $H$ states that can be formed at intermediate states of the reversal process for this range of $\gamma$ 's, as mentioned before. To elucidate the origin of these features, 100 additional different runs were performed for $\gamma=0.035$ by changing the initial seed each time. Results shown in Fig. 4 reveal that all the hysteresis loops without exception, and under the same simulation conditions, fall into four well-defined categories or paths with different probabilities of occurrence. A more detailed analysis allows us to identify the occurrence of two different switching modes along the decreasing or increasing field branches, namely, one with lower coercivity $(Q 1)$ and another with higher
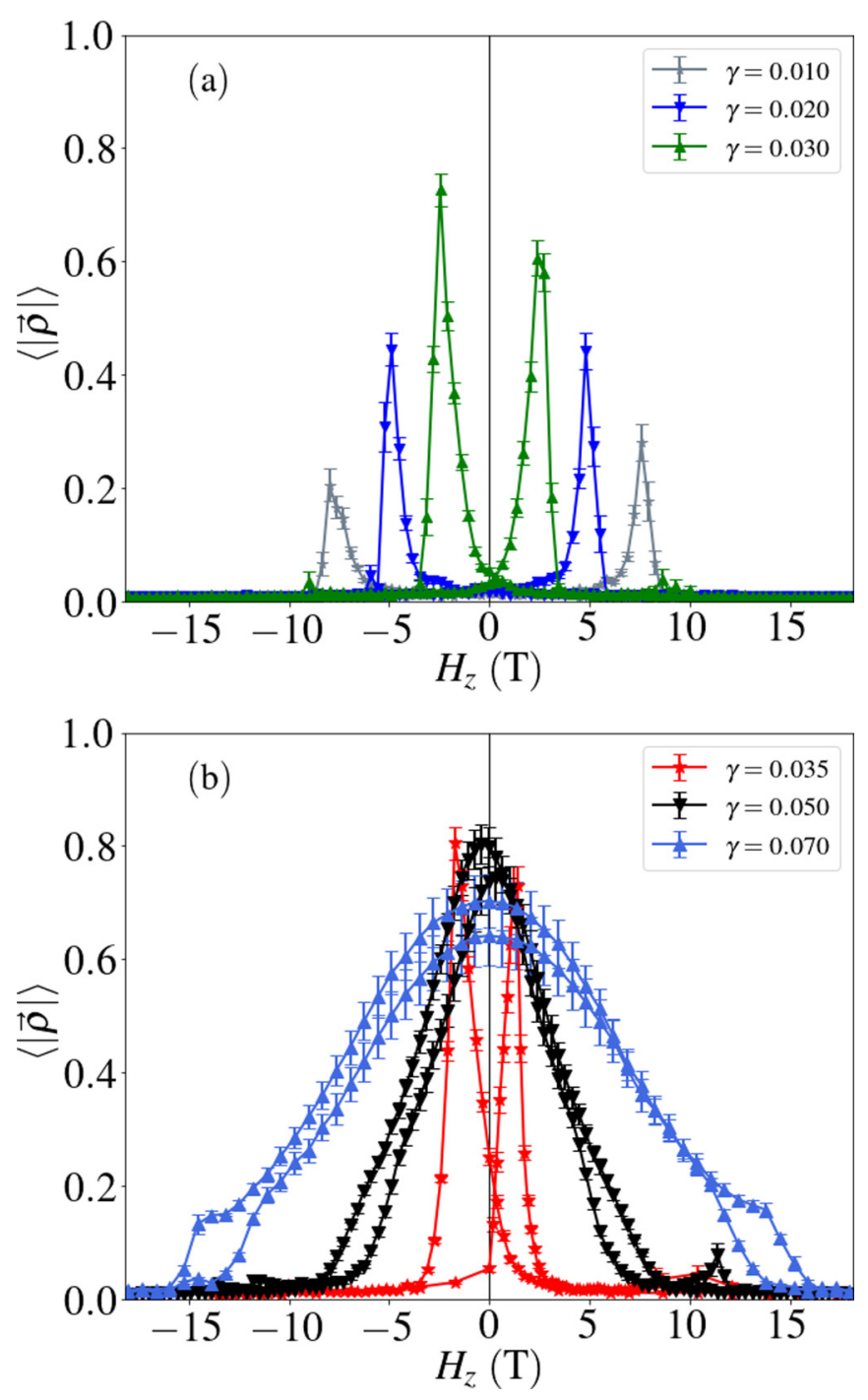

FIG. 3. Same as Fig. 2 but for the average magnitude of the rotational of the magnetization $\langle|\vec{\rho}|\rangle$.

coercivity $(Q 2)$. Thus, the four classes of hysteresis loops can be categorized by combinations of them. Two classes $(Q 1-$ $Q 1, Q 2-Q 2$ ) correspond to symmetric loops [see Figs. 4(a) and 4(d)], while for the other two $(Q 1-Q 2, Q 2-Q 1)$, cycles are asymmetric, resembling those found in exchange-biased systems. Their respective probabilities of occurrence are $86 \%$ $(Q 1-Q 1), 11 \%(Q 1-Q 2), 2 \%(Q 2-Q 1), 1 \%(Q 2-Q 2)$ while the total probabilities per mode or branch are $92.5 \%$ for $Q 1$ and $7.5 \%$ for $Q 2$.

In the $Q 1$ mode, the inversion is nucleated through the formation of $H$ states with the same chirality at the tube ends (see snapshot 1 in Fig. 5, left column). Due to this, the magnetization switching proceeds in a completely coherent fashion through a gradual rotation of the angle $\theta$ at both tube ends, reaching a state at remanence (snapshot 2) that corresponds to an almost perfect vortex.

The formation of this $H$ state and its gradual transition into a vortex is responsible for the low associated coercive field. This behavior is confirmed by the profiles shown in the upper 

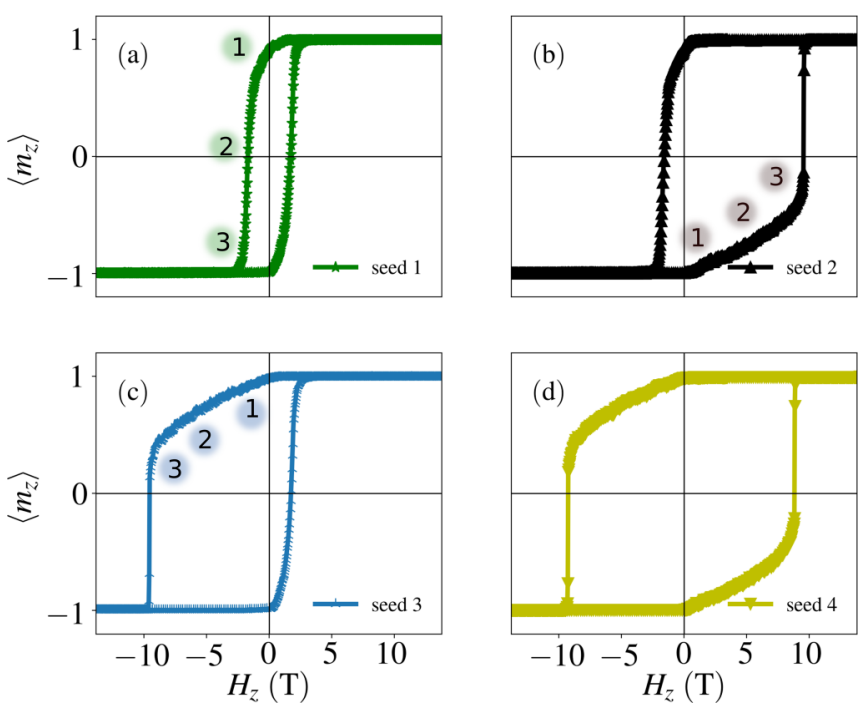

FIG. 4. The four possible paths followed when a $(8,15)$ nanotube with $\gamma=0.035$ is submitted to a hysteresis loop starting from different seeds of the random number generator. The four scenarios are labeled according to the reversal modes followed along the decreasing-increasing field branches as (a) $Q 1-Q 1$, (b) $Q 1-Q 2$, (c) $Q 2-Q 1$, (d) $Q 2-Q 2$, respectively.

left panels of Fig. 5, where for stage 2 , the values $\langle\theta\rangle \approx 90^{\circ}$, $\left\langle m_{z}\right\rangle \approx 0$ and $\left\langle m_{\phi}\right\rangle \approx-1$ are attained.

Unlike this, in the $Q 2$ mode, reversal is started by the formation of $H$ states having opposite chiralities at the tube
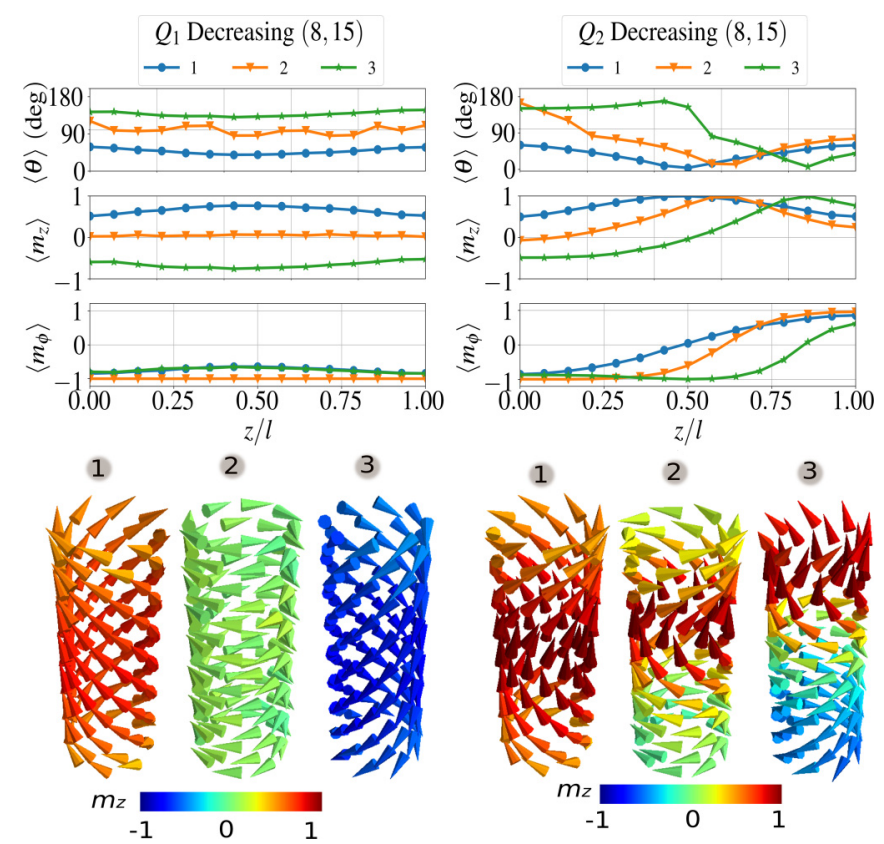

FIG. 5. Magnetic configurations along the hysteresis loops for the different reversal modes displayed in Fig. 4 (left and right columns correspond to panels (a) and (c) of that figure). Upper panels represent the height profiles of the quantities $\langle\theta\rangle,\left\langle m_{z}\right\rangle$, and $\left(<m_{\phi}\right\rangle$ averaged per layer for the tube $(8,15)$ and $\gamma=0.035$, whereas lower ones present snapshots of the spin configurations taken at points labeled in Fig. 4.
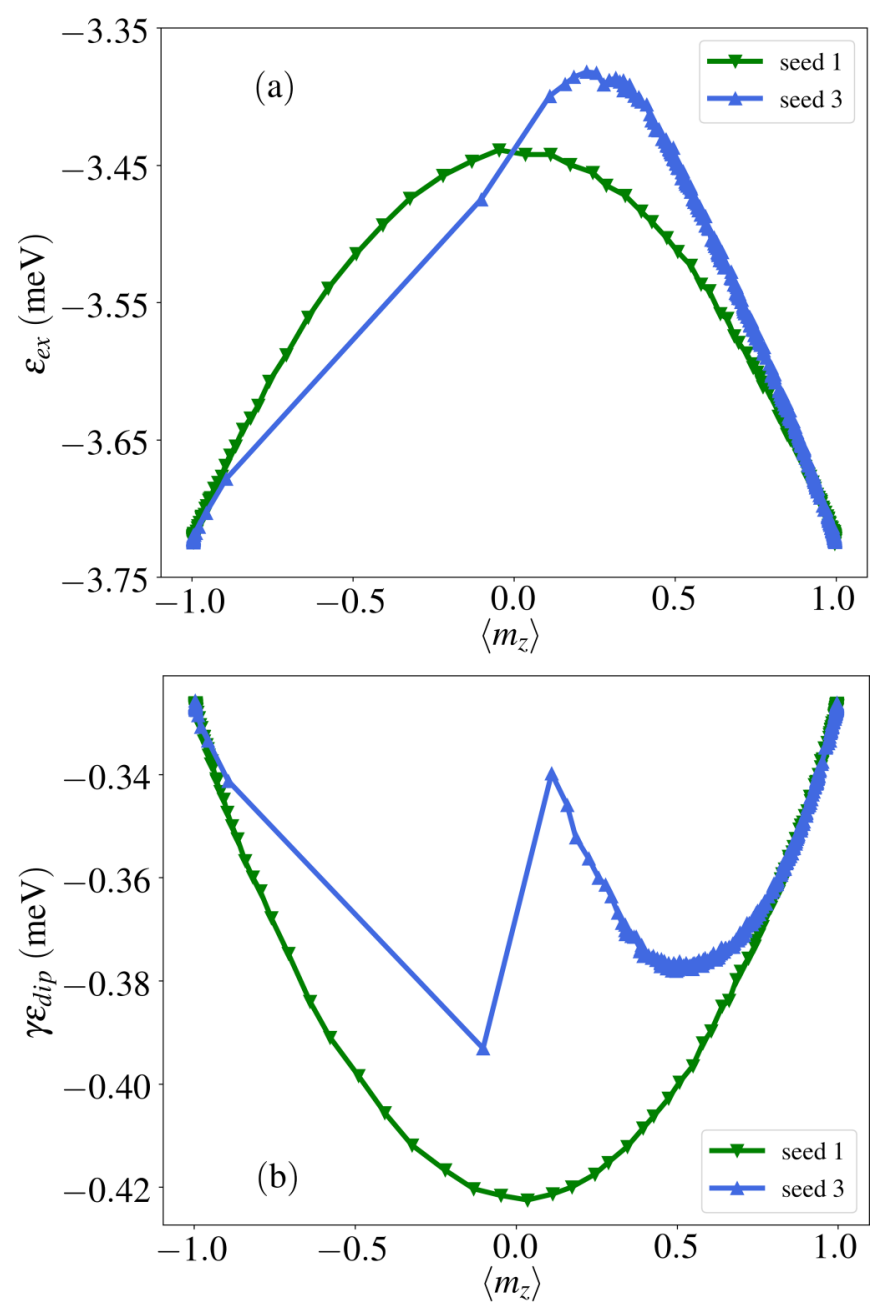

FIG. 6. Dependence of the exchange (a) and dipolar (b) energies of the $(8,15)$ nanotube on its magnetization for the configurations attained along the decreasing field branch of the hysteresis loops shown in Figs. 4(a) and 4(c) (green and blue symbols, respectively) corresponding to seeds 1 and 3 , respectively, in the figure.

ends as can be observed in snapshot 1 in Fig. 5 (right column). As the reversal progresses, $H$ states propagate by forming two domains with opposite directions connected by a domain wall (stage 2 in the right column panels of Fig. 5). This fact is confirmed by the positive and negative values of the $m_{\phi}$ component close to 1 and -1 , respectively, as shown in the respective profiles of the right panels of Fig. 5. The confrontation of opposite chiralities in the central region of the tube makes the system magnetically harder and, therefore, a higher coercive field is obtained.

The occurrence of different paths for reversal can be enlightened by studying the variation of the exchange and dipolar energies along the decreasing field branches of the hysteresis loops of Figs. 4(a) and 4(c). Such variations are shown in Fig. 6 as a function of the corresponding magnetization. For path $Q 1$, while going from saturation to the coercive force, the dipolar energy decreases in a continuous fashion at the expense of an increase in exchange energy, explaining the formation of a $V$ state at a coercive field $H_{c} \approx 1.7 \mathrm{~T}$. On the other hand, path $Q 2$ is characterized by an excursion through 
intermediate states with higher energies and this explains the lower probabilities of occurrence compared to those of path $Q 1$.

Both in $Q 1$ and $Q 2$, reversal starts with identical decrease (increase) of the dipolar (exchange) energy. Differences between paths initiate near remanence with a total average $\left\langle m_{z}\right\rangle \approx 0.8$, from where the $Q 2$ path drives the system through a state with $\left\langle m_{z}\right\rangle \approx 0.5$, characterized by a local minimum in $E_{\text {dip }}$ above the corresponding energy for the $Q 1$ path. Near the coercive force, i.e., $\left\langle m_{z}\right\rangle=0$, the $Q 2$ path displays an abrupt decrease in both $\varepsilon_{\mathrm{ex}}$ and $\varepsilon_{\text {dip }}$ ascribed to the formation of a domain wall connecting $H$ states of opposite chirality. During switching, a confrontation of $H$ states that propagate from the ends takes place in the central region of the tube. One of the $H$ states leaves at the end where it was nucleated and at some point it is followed by the other $H$ state (see snapshot 3 in the right column of Fig. 5).

It is worth mentioning that when dipolar interactions dominate over exchange $\left(\gamma>\gamma^{\star}\right)$, as can be seen in Fig. 2(b), the averaged loops become more tilted with higher closure fields. This is a consequence of the formation of almost perfect $V$ states at remanence, which for this range of $\gamma$ 's are the minimum energy configurations [23]. Two possible reversal modes are also found in this range, again depending on the formation of states at the ends with equal or opposite chiralities in the early stages of reversal. However, now one of the modes ( $Q 1$ : low coercivity) has a negligible coercive field (not shown) and it corresponds to the formation of states for which vorticity varies monotonically with a maximum close to 1 . In contrast, the high coercivity mode $(Q 2)$ is characterized by greater values of the coercive field compared to those of their low- $\gamma$ counterparts.

\section{B. Tailoring reversal modes}

To verify the reproducibility of the paths observed in the $Q 1$ and $Q 2$ modes, we have examined the possibility to induce the magnetization reversal by either of the two modes in a controlled manner by preparing the tubes in two initial configurations near the remanence. Such as-prepared states consist in a central region where spins are aligned along the tube length $\left(\langle\theta\rangle=0^{\circ},\left\langle m_{z}\right\rangle=1\right.$ and $\left.\left\langle m_{\phi}\right\rangle=0\right)$ and a helical order in the last two layers at both ends of the tube. In one case, the two ends have the same chirality $\left[\langle\theta\rangle=45^{\circ}\right.$, $\left\langle m_{z}\right\rangle=\cos \left(45^{\circ}\right)$ and $\left.\left\langle m_{\phi}\right\rangle=-\sin \left(45^{\circ}\right)\right]$, i.e., with the same sign of $\left\langle m_{\phi}\right\rangle$ at the ends, and in the other case, opposite chiralities are manufactured, i.e., $\left(\langle\theta\rangle=45^{\circ},\left\langle m_{z}\right\rangle=\cos \left(45^{\circ}\right)\right.$ and $\left.\left\langle m_{\phi}\right\rangle= \pm \sin \left(45^{\circ}\right)\right)$. Due to the cylindrical symmetry, both states result in the same total magnetization $[\langle|\vec{m}|\rangle=$ $\left.\left\langle m_{z}\right\rangle=0.92,\left\langle m_{x}\right\rangle=\left\langle m_{y}\right\rangle=0\right]$ and the same energy $(\langle E\rangle=$ $-4.5 \mathrm{meV}$ per spin).

These initial states were then allowed to evolve under the same conditions along the decreasing field branch of the hysteresis loop. Results are shown in Fig. 7 and the respective initial as-prepared states along with some intermediate spin configurations during magnetization reversal are shown in Fig. 8. Starting from the state with the same chirality at both ends, the system always evolves through the $Q 1$ inversion mode of lower coercivity, i.e., $P(Q 1)=100 \%$. However, starting from the configuration with opposite chiralities, the

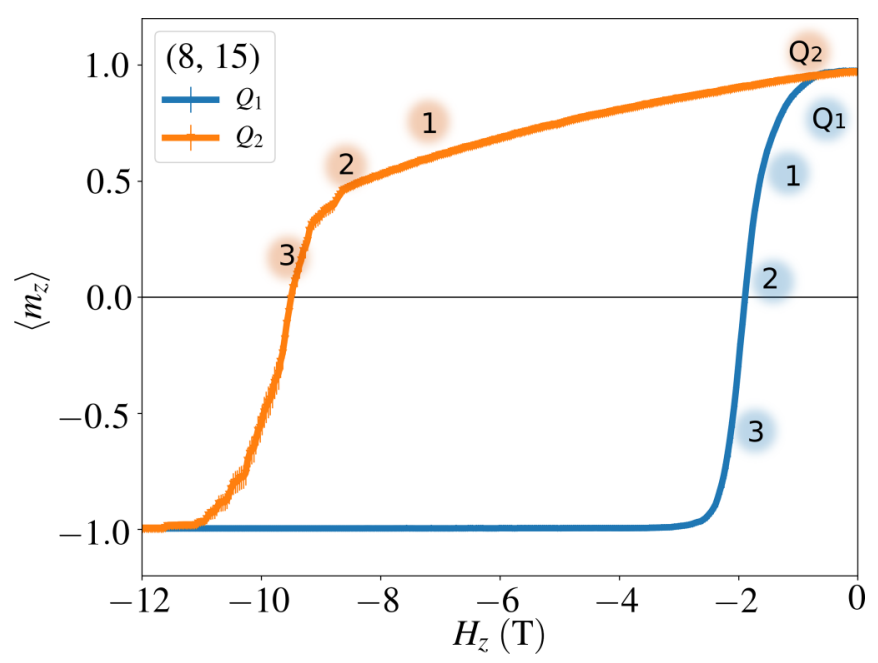

FIG. 7. Decreasing field branch of the hysteresis loops for a $(8,15)$ tube with $\gamma=0.035$ for two states initially prepared with $H$ states of the same $(Q 1)$ or opposite $(Q 2)$ chiralities at the tube ends.

probability to follow the $Q 2$ mode is $P(Q 2)=85 \%$, whereas for the $Q 1$ mode it is $P(Q 1)=15 \%$. These features reveal the subtleties and complexity of the energy landscape and the differences in energy between $Q 1$ and $Q 2$ modes. Based on the results obtained, we have also investigated the effect that the tube length plays in the observed metastability of the reversal modes without changing $\gamma$, i.e., for $\gamma=0.035$. For this purpose, additional simulations were performed for
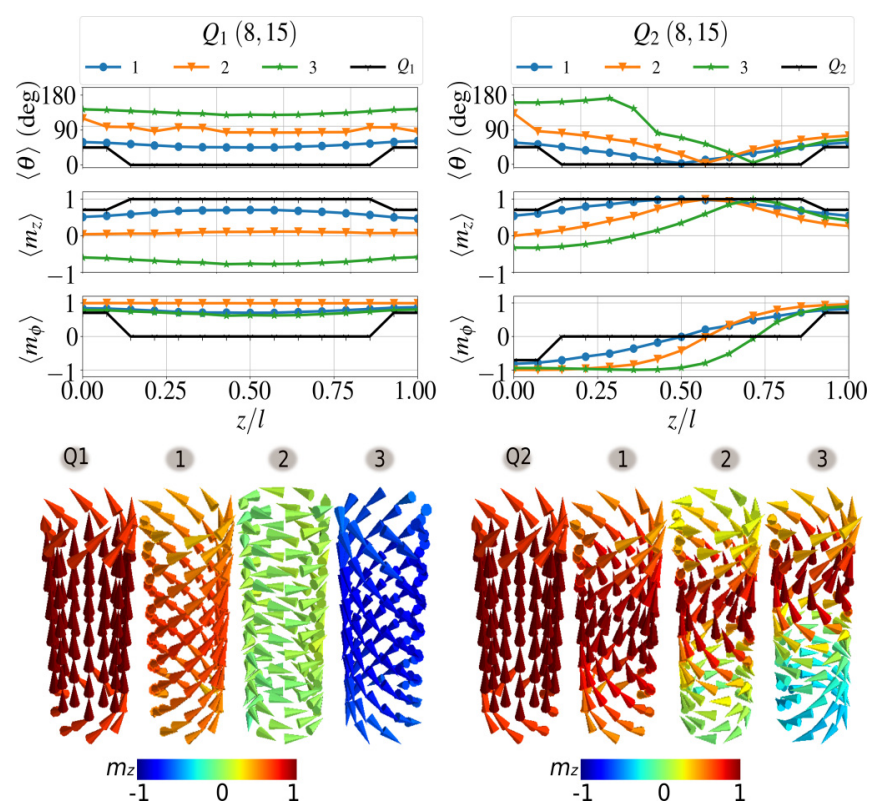

FIG. 8. Magnetic configurations during magnetization reversal when starting from an initial state $Q 1(Q 2)$ prepared with the same (opposite) chirality at the ends of the tube. Upper panels represent the height profiles of the quantities $\langle\theta\rangle,\left\langle m_{z}\right\rangle$, and $\left\langle m_{\phi}\right\rangle$ averaged per layer for the tube $(8,15)$ and $\gamma=0.035$, whereas lower ones present snapshots of the spin configurations taken at the points labeled in Fig. 7. Profiles of the initial remanence prepared state are included for comparison. 

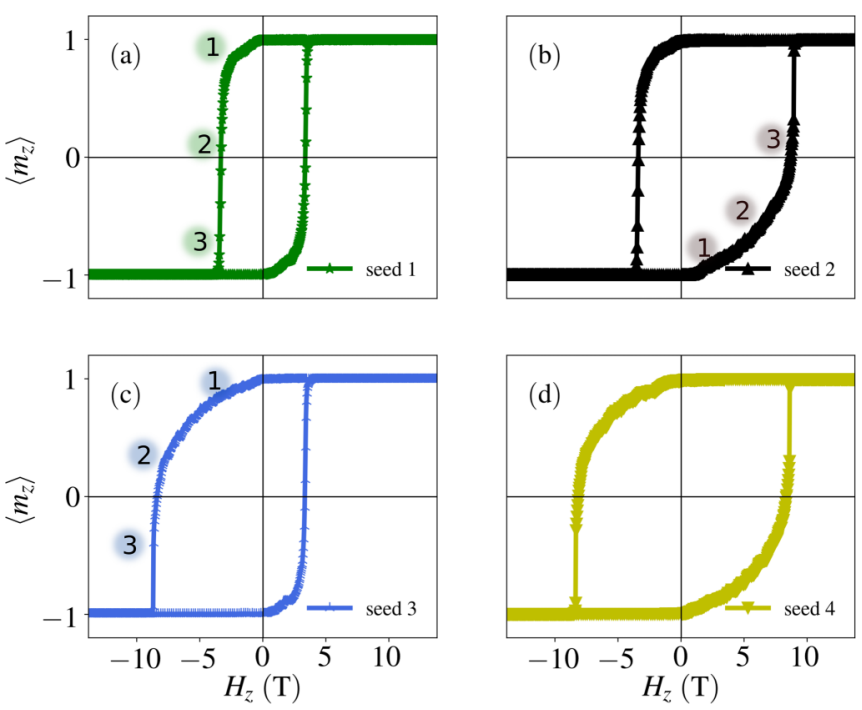

FIG. 9. The four possible paths followed when a $(8,20)$ nanotube with $\gamma=0.035$ is submitted to a hysteresis loop simulated starting from different seeds of the random number generator. The four cases are named according to the reversal modes followed along the decreasing-increasing field branches: (a) $Q 1-Q 1$, (b) $Q 1-Q 2$, (c) $Q 2-Q 1$, (d) $Q 2-Q 2$.

two other tubes, namely, $(8,20)$ and $(8,8)$ tubes. In the former, results evidence the same phenomenology of the $(8,15)$ tube (see Figs. 9 and 10), although now the coercive field of the $Q 1$ mode has increased whereas that of the $Q 2$ mode has decreased compared to the respective values of the $(8,15)$ tube.
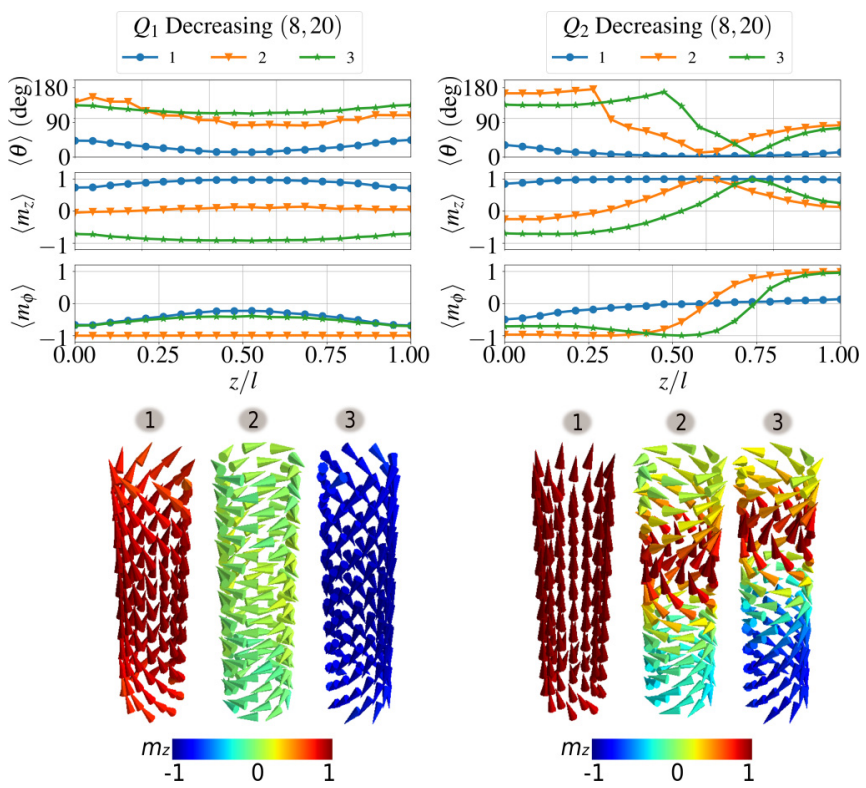

FIG. 10. Magnetic configurations along the hysteresis loops for the different reversal modes displayed in Fig. 9 (left and right columns correspond to panels (a) and (c) of the figure). Upper panels represent the height profiles of the quantities $\langle\theta\rangle,\left\langle m_{z}\right\rangle$, and $\left\langle m_{\phi}\right\rangle$ averaged per layer for the tube $(8,20)$ and $\gamma=0.035$, whereas the lower ones are snapshots of the spin configurations taken at points labeled in Fig. 9.
Moreover, differences can be observed in the probabilities of occurrence per category, which are now $31 \%(Q 1-Q 1)$, $26 \%(Q 1-Q 2), 26 \%(Q 2-Q 1), 17 \%(Q 2-Q 2)$ with total probabilities per single mode of $P(Q 1)=57 \%$ and $P(Q 2)=$ $43 \%$. Notice that these last probabilities are closer each other, with a trend to equiprobability, which contrasts with the results for the $(8,15)$ tube, where $P(Q 1)=92.5 \%$ and $P(Q 2)=7.5 \%$.

On the other hand, when performing the simulations with the two initial as-prepared states, i.e., with the same $(Q 1)$ or opposite $(Q 2)$ chiralities at the ends, by following the same procedure of the $(8,15)$ tube, we now obtain a low-coercive reversal mode probability $P(Q 1)=100 \%$ when starting from remanence $Q 1$ and a high-coercive reversal mode probability $P(Q 2)=100 \%$ when starting from remanence $Q 2$. In this way, we have been able to demonstrate that, at least for nanotubes with a higher aspect ratio $N_{z} / N$, under tuning of the remanence states $Q 1$ or $Q 2$, the coercive force can be completely tailored to a low or high value, respectively.

Now, if we modify the degree of competition between energies, by tuning the $\gamma$ value to the respective $\gamma^{\star} \approx 0.045$ of a $(8,20)$ tube, for which $H$ states are energetically more favorable in the zero-field magnetic-phase diagram [23], the total probabilities per single mode become almost equiprobable with $P(Q 1)=52.5 \%$ and $P(Q 2)=47.5 \%$. Moreover, those remanence states having opposite chiralities in the processes $Q 1-Q 2$ and $Q 2-Q 1$ result in having very different probabilities of occurrence if the number of layers is an odd number. This fact makes the remanence spin configuration asymmetric relative to the field direction, i.e., the system is not invariant under field reversal. This is the reason why for the $(8,15)$ tube the probabilities $P(Q 1-Q 2)=11 \%$ and $P(Q 2-Q 1)=2 \%$ were obtained. Contrary to this, if the number of layers is an even number, the remanence spin configuration is symmetric and the probabilities $P(Q 1-Q 2)$ and $P(Q 2-Q 1)$ tend to be equal as indeed observed for the $(8,20)$ tube. To verify this issue, additional simulations were performed for a tube $(8,14)$, very close to the $(8,15)$ tube. In such a case, it turns out that $P(Q 1-Q 2)=P(Q 2-Q 1)=2 \%$. In this respect, it is easy to notice that if the number of layers is odd, in a state of opposite chiralities, there will be an unbalance between the number of layers with a given chirality and the number of layers with the opposite one. The asymmetry becomes more relevant depending on the decreasing or increasing field direction. Such unbalance is negligible in scenarios of tubes with an even number of layers.

Regarding the $(8,8)$ nanotube, hysteresis loops (averaged over 100 realizations) and the respective vorticity curves were also studied, see Fig. 11. In this case, tilted hysteresis loops for $\gamma=0.035$ do not reveal the appearance of the two modes. Despite the existence of small regions in the cycle near saturation with a greater irreversibility, the associated coercivity is very small, leading to the occurrence of one single $(Q 1)$ mode. Here, the short length of the tube makes it unable to harbor the vortex domain wall of opposite chiralities, suppressing in this way the high-coercivity $(Q 2)$ mode. Thus, only the low-coercivity $(Q 1)$ mode subsists.

These observations are in agreement with the fact that reversal modes in magnetic nanotubes are strongly dependent on geometrical factors, particularly the aspect ratios, as has 


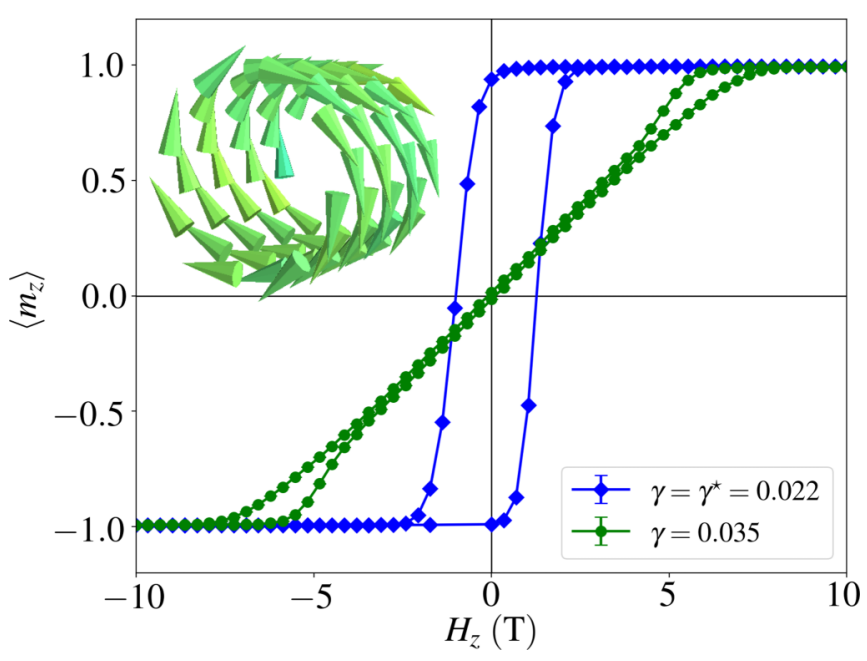

FIG. 11. Hysteresis loops of a $(8,8)$ tube for two different values of $\gamma$. The spin configuration corresponds to the single kind of remanence state with the same chiralities at the ends present in this system.

been already published [30,31,36], so differences when the aspect ratios change are expected even if the same initial states are considered. Finally, for a different competition degree, namely, $\gamma=\gamma^{\star} \approx 0.022$, also one single $Q 1$ mode subsists, but now the magnetization reversal occurs practically in one single step in a very narrow region in the vicinity of the coercive field. Contrary to this, for $\gamma=0.035$, magnetization reversal occurs accompanied by circular states $\left(\left\langle\rho_{z}\right\rangle \neq 0\right)$ persistent along most of the cycle.

\section{Micromagnetic simulations}

To further investigate the possibility of observing the above-mentioned phenomenology in tubes of greater dimensions in the range of tens of nanometers, closer to those of tubes experimentally synthesized, a micromagnetic approach has been conducted. For this purpose, tubes having the same aspect ratio to that of the $(8,20)$ tube considered in the MC simulations have been modeled.

We would like to stress that it is not possible to establish a one-to-one correspondence between an atomistic model for which we consider a layer of spins rolled to form a monoatomic thick tube with a micromagnetic model for which the tube thickness has to be finite. Therefore, even though the $\gamma$ parameter cannot be mapped exactly to a micromagnetic equivalent, we can have an estimation of the $\gamma$ parameter for the continuous model used in micromagnetics. To do so, we approximate the volume density of the energies $E_{\text {ex }} \approx$ $A / a^{2}$ and $E_{\mathrm{mag}} \approx \mu_{0} M_{s}^{2} / 2$ and estimate their ratio as $\gamma \simeq$ $E_{\mathrm{mag}} / E_{\mathrm{ex}}, a$ being the lattice parameter. With the values for FeCo mentioned in Sec. II B, $\gamma=0.016$, while a value of $\gamma$ close to the $\gamma^{\star} \approx 0.045$ of the $(8,20)$ tube can be obtained by choosing, for example, $M_{s}=2 \times 10^{6} \mathrm{~A} / \mathrm{m}, A=10 \times 10^{-12}$ $\mathrm{J} / \mathrm{m}$ and $a=0.3 \mathrm{~nm}$, which are within the typical values for ferromagnets. Micromagnetic calculations were performed for these last values.

In this approach, we have ensured first that, using the energy minimization procedure via OOMMF at $T=0$, ground-
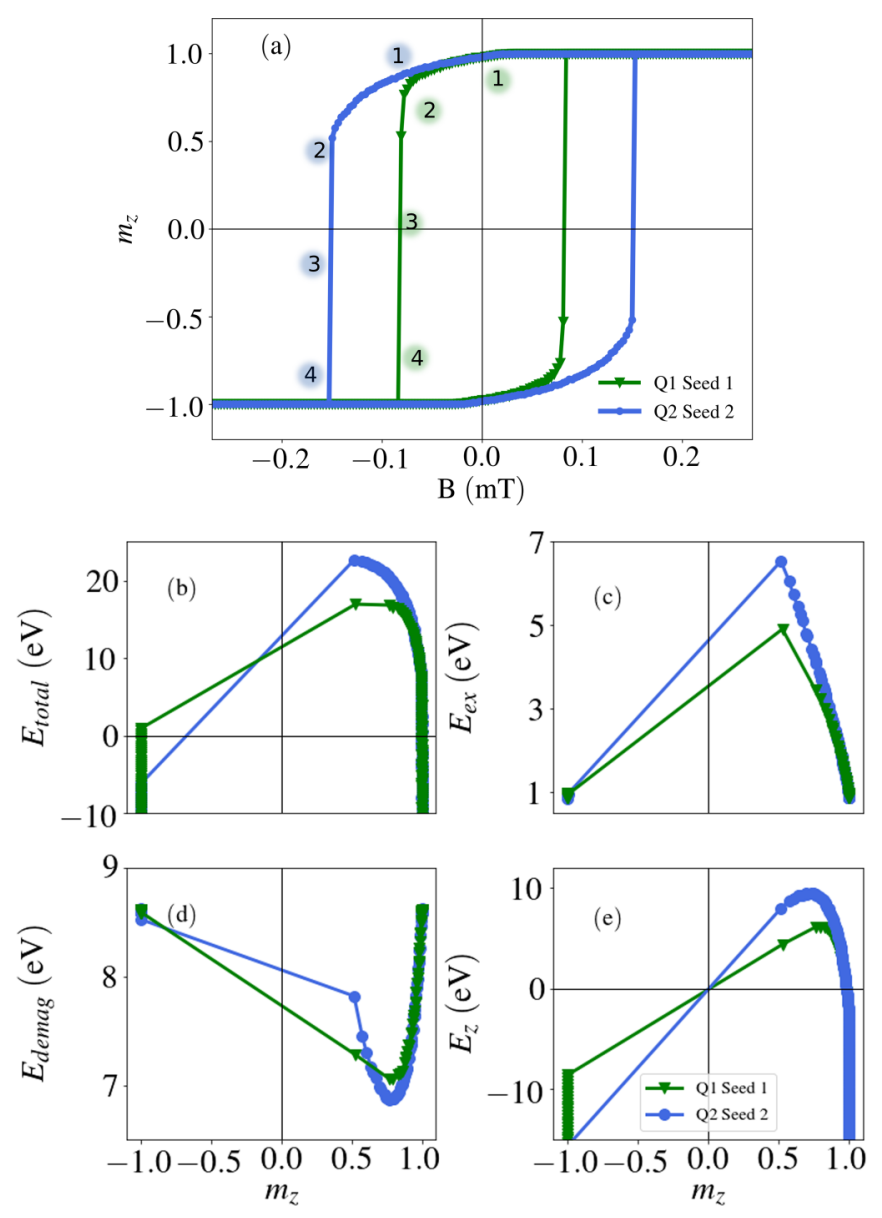

FIG. 12. (a) Hysteresis loops simulated with OOMMF at $T=$ $4 \mathrm{~K}$ starting from saturation for two different seeds for the random number used to generate the stochastic thermal fields. Lower panels display the dependence of the total (b), exchange (c), dipolar (d), and Zeeman (e) energies of the nanotube on its magnetization for the configurations attained along the decreasing field branch of the hysteresis loops shown in (a).

state configurations similar to those obtained by $\mathrm{MC}$ are obtained for the values of $A$ and $M_{s}$ mentioned above. In particular, by starting from a FM configuration, results show that, after some transient time, the value of the energy is stabilized when the magnetic configuration reaches the equivalent $H$ state. Thus, once it was established that circular states are achievable in micromagnetic simulations, hysteresis loops were computed.

In analogy with the MC simulations, the hysteresis loops were repeated using different random seeds for the generation of the stochastic thermal fields at temperature $T=4 \mathrm{~K}$. The intention is to give the system a chance to follow different paths through the energy landscape at each field stage, so different metastable states, which can be very close in energy but have different microstates, can be reached along the hysteresis loop. This is reasonable as long as a hysteresis loop is a nonequilibrium curve. Thus, as can be observed in the simulated hysteresis loops of Fig. 12, two well-distinguished modes $(Q 1$ and $Q 2)$ for magnetization reversal with two different coercivities were also obtained, in analogy with the ones obtained via $\mathrm{MC}$ for the $(8,15)$ and $(8,20)$ tubes. 


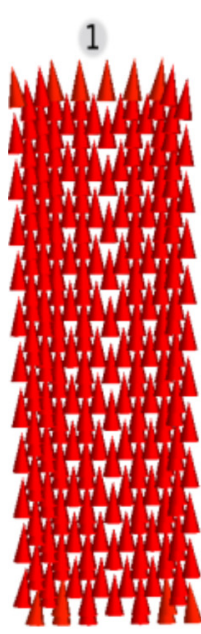

2
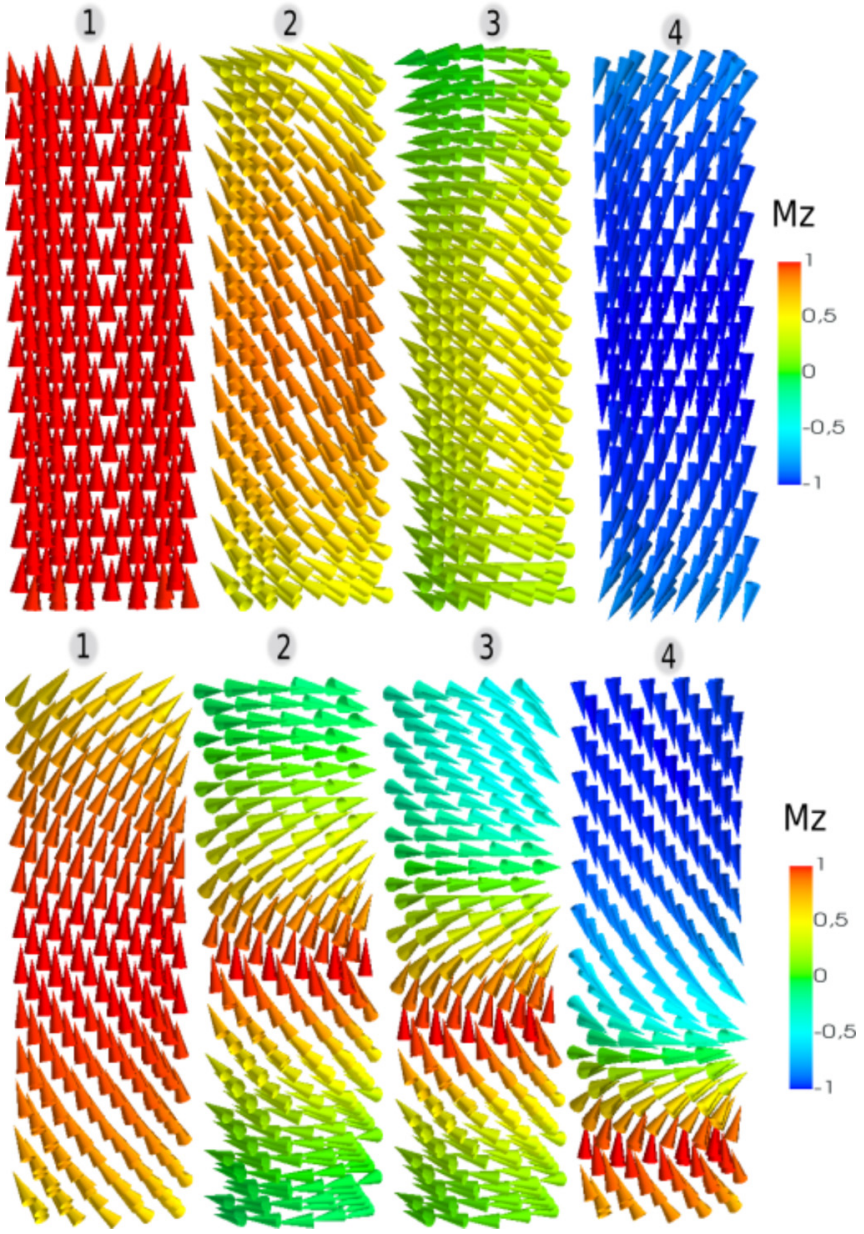

FIG. 13. Snapshots of the spin configurations in the reversal modes $Q 1$ (upper panel) and $Q 2$ (lower panel) of the hysteresis loops obtained from micromagnetic simulations. Numerical labels correspond to those states shown in Fig. 12.

Similarly, tracking of the spin configurations along the decreasing field branches of the loops allows us to identify both switching modes in terms of a dual mechanism. The $Q 1$ mode (obtained for a particular random seed) follows an intermediate stage characterized by $H$ states at the ends of the tube with the same chirality giving rise to a low coercive field. In contrast, when a different random seed is used, the $Q 2$ mode is observed, characterized by intermediate stages with helicities of opposite chirality that give rise to a higher coercive field. Snapshots of these two reversal modes are shown in Fig. 13.

The variations of the energy contributions along the hysteresis loops given in the lower panels of Fig. 12 have a similar behavior to those obtained by MC simulations in Fig. 6 . In particular, energies for both modes are almost identical at high fields and they bifurcate near the remanence. Also, beyond that point, in both cases the mode with opposite chiralities makes an excursion to higher exchange energies [Fig. 12(c)], altough in the MC case the variation is more progressive, in agreement with the smoother variation of the magnetization near the coercive field [compare Figs. 6(a) and 6(d) to Fig. 12(a)]. A similar observation is applicable when

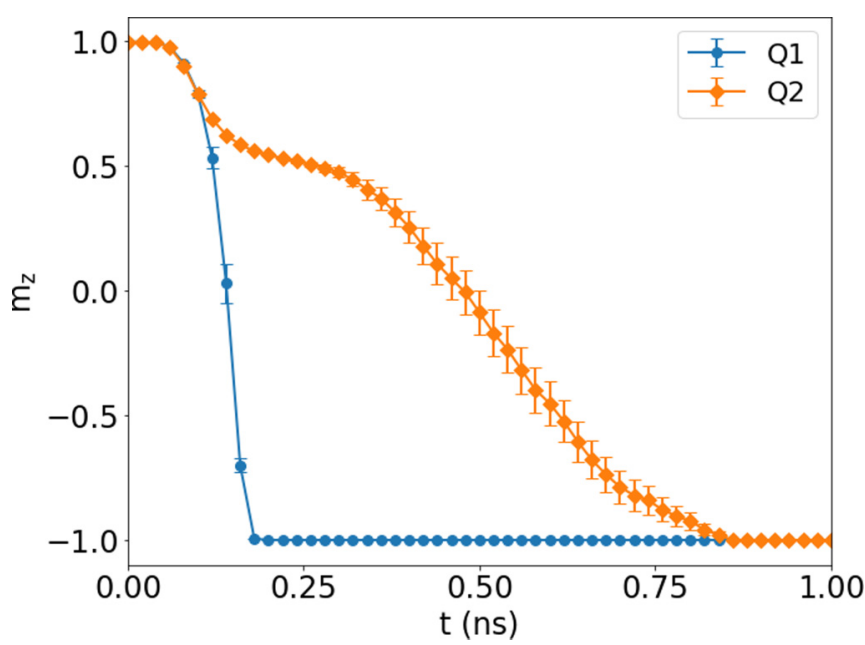

FIG. 14. Magnetization reversal at $T=4 \mathrm{~K}$. Steps of 1 ps were considered for time evolution. $H=0.9 H_{c}$. Error bars were computed over 50 runs starting from different random seeds.

comparing dipolar energies in Fig. 6(b) to demagnetizing ones in Fig. 12(d), although in the latter case the differences between both modes are not as pronounced as in the MC simulations. Similar jumps in energy have also been reported to occur during the reversal of vortices in micromagnetic simulations of nanodots [50].

\section{Thermal effects}

To evaluate the thermal effects in the micromagnetic approach upon the occurrence of the dual inversion modes, we have examined the time evolution of the magnetization at a finite temperature $T=4 \mathrm{~K}$, and for an external applied field below and close to the largest coercive force, namely, $H=0.9 H_{c}$ (coercive field of the Q2 mode), by starting from a saturated initial configuration for different seeds. Results, shown in Fig. 14, are the averages over 50 runs.

As can be observed, the two magnetization reversal modes $Q 1$ and $Q 2$ are still present and they are well differentiated. The differences between the characteristic reversal times for both modes are clearly linked to the different paths followed through the energy landscape. Of course, depending on temperature, differences regarding the probabilities of occurrence per mode are expected to change with respect to the atomistic simulations.

\section{DISCUSSION AND CONCLUSIONS}

We have shown that the occurrence of both helical $(H)$ and vortex $(V)$ states during the magnetization reversal of nanotubes is dictated by $\gamma$, the dipolar to exchange energy ratio. For a certain range of $\gamma$ 's, the results of MC atomistic simulations have demonstrated that different reversal modes can occur along the hysteresis loop as a consequence of the high degree of metastability of the $H$ states that facilitates different paths through the energy landscape when varying the magnetic field. In agreement with our results, recent experimental works on individual magnetic nanotubes $[32,33]$ have also evidenced that short $\mathrm{FeCoB}$ nanotubes $(0.6 \mu \mathrm{m}$ 
long, $300 \mathrm{mn}$ in diameter), with similar aspect ratios as the ones studied here, can be found in mixed states with end vortices of opposing or matching circulations depending on the magnetic history or experiment repetition. Moreover, the comparison of cantilever magnetometry [33] with micromagnetic simulations showed that reversal initiated with matching vortices is correlated to lower energies and smoother energy variations than for opposing vortices. It is worth mentioning also that in Ref. [21] a high degree of data scattering in the two branches of the hysteresis loop as well as a small exchange-bias-like effect in an individual pure Ni tube was observed, which we assume could be attributed to the different reversal possibilities shown in this work.

Our results allow us to conclude that the reversal modes initiated by vortices with same chirality have coercive fields lower than the mode with opposite chiralities, since in this case the central region of the tube has to face the merging of opposite directions of helicity due to the formation of a domain wall. Our proposal to induce the reversal through the $Q 1$ or $Q 2$ modes, based on controlling the initial chirality at the tube ends, would allow us to use a unique tube as a soft or hard material without changing its composition. In fact, the experimental study of Ref. [33] has already suggested that control over relative chirality can be achieved introducing structural asymmetries at the nanotube ends, but we have shown that this might be achieved also without modifying the tube structure.

Regarding vorticity control in magnetic tubes without modifying their structure, some previous works can be considered as a step in such direction. Namely, (i) by using magnetic field pulses along the symmetry-axis of the tube [51], (ii) by means of the injection of electric current pulses along the symmetry axis of the tube [52], and (iii) by injection of small electric currents flowing through the nanotube with a circular magnetic field applied at the middle of the tube [53] as a way to stabilize a vortex state, which can eventually be used as a starting state for an inversion process.

Finally, apart from the scenario of having clusters or nanoparticles grafted or attached to the surface of a tube as a template where dipolar interactions can gain importance, it is also viable to pose as an alternative the use of SMMs, which can be assembled, by using coordination chemistry, into1D, 2D, and 3D networks [54]. The experimental challenge would be to get a 2D network of SMMs in a cylindrical arrangement an estimate of the $\gamma$ values involved in this kind of systems can be assessed by considering, for instance, the FM $\mathrm{Mn}_{4}$ molecular magnet [55] with an average magnetic moment between $\mathrm{Mn}^{+2}$ and $\mathrm{Mn}^{+3}$ of $4.15 \mu_{B}$, an average distance of $3.31 \AA$, and a FM exchange interaction of $6.5 \mathrm{~K}$. These values yield $\gamma=0.046$, placing us in the range where dual reversal modes may appear.

Although in this paper we have limited our study to tubes of reduced dimensions, we have given proof that our conclusions are not peculiar to the range of sizes studied. Preliminary results to be shown in a forthcoming publication indicate that a similar phenomenology can be observed for longer or wider tubes under certain conditions of competition according to $\gamma$.

\section{ACKNOWLEDGMENTS}

Ò.I. acknowledges financial support form the Spanish MINECO (No. MAT2015-68772 and No. PGC2018-097789B-I00), Catalan DURSI (No. 2017SGR0598) and European Union FEDER Funds (una manera de hacer Europa), also CSUC for supercomputer facilities. H.D.S and J.R. acknowledge financial support from the Colciencias Beca de Doctorados Nacionales, ConvocatoriaNo. 727, Project No. CODIUdeA 2017-16253. J.R. acknowledges UdeA for the exclusive dedication program.
[1] M. Staňo and O. Fruchart, Magnetic nanowires and nanotubes, in Handbook of Magnetic Materials, edited by E. Brück (Elsevier, the Netherlands, 2018), Vol. 27, Chap. 3, pp. 155-267.

[2] Y. Ye and B. Geng, Magnetic Nanotubes: Synthesis, Properties, and Applications, Crit. Rev. Solid State Mater. Sci. 37, 75 (2012).

[3] K. Nielsch, F. J. Castaño, C. A. Ross, and R. Krishnan, Magnetic properties of template-synthesized cobalt polymer composite nanotubes, J. Appl. Phys. 98, 034318 (2005).

[4] X. F. Han, S. Shamaila, R. Sharif, J. Y. Chen, H. R. Liu, and D. P. Liu, Structural and magnetic properties of various ferromagnetic nanotubes, Adv. Mater. 21, 4619 (2009).

[5] Y. C. Sui, R. Skomski, K. D. Sorge, and D. J. Sellmyer, Nanotube magnetism, Appl. Phys. Lett. 84, 1525 (2004).

[6] Y. Xu, D. S. Xue, J. L. Fu, D. Q. Gao, and B. Gao, Synthesis, characterization and magnetic properties of Fe nanotubes, J. Phys. D: Appl. Phys. 41, 215010 (2008).

[7] M. Daub, M. Knez, U. Goesele, and K. Nielsch, Ferromagnetic nanotubes by atomic layer deposition in anodic alumina membranes, J. Appl. Phys. 101, 1 (2007).

[8] C. Garcia, W. O. Rosa, J. Garcia, V. M. Prida, B. Hernando, J. A. López, P. Vargas, and C. A. Ross, Magnetization reversal in radially distributed nanowire arrays, J. Phys. Chem. C 122, 5124 (2018).

[9] R. P. Cowburn and M. E. Welland, Room temperature magnetic quantum cellular automata, Science 287, 1466 (2000).

[10] S. S. P. Parkin, M. Hayashi, and L. Thomas, Magnetic domainwall racetrack memory, Science 320, 190 (2008).

[11] J. Alonso, H. Khurshid, V. Sankar, Z. Nemati, M. H. Phan, E. Garayo, J. A. García, and H. Srikanth, FeCo nanowires with enhanced heating powers and controllable dimensions for magnetic hyperthermia, J. Appl. Phys. 117, 17D113 (2015).

[12] J. A. Fernandez-Roldan, D. Serantes, R. P. del Real, M. Vazquez, and O. Chubykalo-Fesenko, Micromagnetic evaluation of the dissipated heat in cylindrical magnetic nanowires, Appl. Phys. Lett. 112, 212402 (2018).

[13] X. Chen, R. Klingeler, M. Kath, A. A. E. Gendy, K. Cendrowski, R. J. Kalenczuk, and E. Borowiak-palen, Magnetic silica nanotubes: Synthesis, drug release, and feasibility for magnetic hyperthermia, ACS Appl. Mater. Interfaces 4, 2303 (2012).

[14] H. D. Salinas and J. Restrepo, Influence of the competition between dipolar and exchange interactions on the magnetic 
structure of single-wall nanocylinders. Monte Carlo simulation, J. Supercond. Novel Magn. 25, 2217 (2012).

[15] A. Chen, N. Usov, J. Blanco, and J. Gonzalez, Equilibrium magnetization states in magnetic nanotubes and their evolution in external magnetic field, J. Magn. Magn. Mater. 316, e317 (2007).

[16] A. P. Chen, K. Y. Guslienko, and J. Gonzalez, Magnetization configurations and reversal of thin magnetic nanotubes with uniaxial anisotropy, J. Appl. Phys. 108, 083920 (2010).

[17] A.-P. Chen, J. M. Gonzalez, and K. Y. Guslienko, Magnetization configurations and reversal of magnetic nanotubes with opposite chiralities of the end domains, J. Appl. Phys. 109, 073923 (2011)

[18] J. Escrig, P. Landeros, D. Altbir, E. Vogel, and P. Vargas, Phase diagrams of magnetic nanotubes, J. Magn. Magn. Mater. 308, 233 (2007).

[19] N. Biziere, C. Gatel, R. Lassalle-Balier, M. C. Clochard, J. E. Wegrowe, and E. Snoeck, Imaging the fine structure of a magnetic domain wall in a Ni nanocylinder, Nano Lett. 13, 2053 (2013).

[20] D. P. Weber, D. Rüffer, A. Buchter, F. Xue, E. Russo-Averchi, R. Huber, P. Berberich, J. Arbiol, A. Fontcuberta i Morral, D. Grundler, and M. Poggio, Cantilever magnetometry of individual Ni nanotubes, Nano Lett. 12, 6139 (2012).

[21] A. Buchter, J. Nagel, D. Rüffer, F. Xue, D. P. Weber, O. F. Kieler, T. Weimann, J. Kohlmann, A. B. Zorin, E. RussoAverchi, R. Huber, P. Berberich, A. Fontcuberta i Morral, M. Kemmler, R. Kleiner, D. Koelle, D. Grundler, and M. Poggio, Reversal Mechanism of an Individual Ni Nanotube Simultaneously Studied by Torque and Squid Magnetometry, Phys. Rev. Lett. 111, 067202 (2013).

[22] A. Yamasaki, W. Wulfhekel, R. Hertel, S. Suga, and J. Kirschner, Direct Observation of the Single-Domain Limit of fe Nanomagnets by Spin-Polarized Scanning Tunneling Spectroscopy, Phys. Rev. Lett. 91, 127201 (2003).

[23] H. D. Salinas, J. Restrepo, and Ò. Iglesias, Change in the magnetic configurations of tubular nanostructures by tuning dipolar interactions, Sci. Rep. 8, 10275 (2018).

[24] R. Streubel, F. Kronast, P. Fischer, D. Parkinson, O. G. Schmidt, and D. Makarov, Retrieving spin textures on curved magnetic thin films with full-field soft $x$-ray microscopies, Nat. Commun. 6, 7612 (2015).

[25] A. Buchter, R. Wölbing, M. Wyss, O. F. Kieler, T. Weimann, J. Kohlmann, A. B. Zorin, D. Rüffer, F. Matteini, G. Tütüncüoglu, F. Heimbach, A. Kleibert, A. Fontcuberta i Morral, D. Grundler, R. Kleiner, D. Koelle, and M. Poggio, Magnetization reversal of an individual exchange-biased permalloy nanotube, Phys. Rev. B 92, 214432 (2015).

[26] C. Donnelly, M. Guizar-Sicairos, V. Scagnoli, S. Gliga, M. Holler, J. Raabe, and L. J. Heyderman, Three-dimensional magnetization structures revealed with $\mathrm{x}$-ray vector nanotomography, Nature (London) 547, 328 (2017).

[27] M. Zimmermann, T. N. G. Meier, F. Dirnberger, A. Kákay, M. Decker, S. Wintz, S. Finizio, E. Josten, J. Raabe, M. Kronseder, D. Bougeard, J. Lindner, and C. H. Back, Origin and manipulation of stable vortex ground states in permalloy nanotubes, Nano Lett. 18, 2828 (2018).

[28] D. Vasyukov, L. Ceccarelli, B. G. M. Wyss, A. Schwarb, A. Mehlin, N. Rossi, G. Tütüncüoglu, F. Heimbach, R. R.
Zamani, A. Kovács, A. F. i Morral, D. Grundler, and M. Poggio, Imaging stray magnetic field of individual ferromagnetic nanotubes, Nano Lett. 18, 964 (2018).

[29] A. L. Balk, I. Gilbert, R. Ivkov, J. Unguris, and S. M. Stavis, Bubble Magnetometry of Nanoparticle Heterogeneity and Interaction, Phys. Rev. Appl. 11, 061003 (2019).

[30] R. Sharif, S. Shamaila, M. Ma, L. D. Yao, R. C. Yu, X. F. Han, and M. Khaleeq-ur Rahman, Magnetic switching of ferromagnetic nanotubes, Appl. Phys. Lett. 92, 032505 (2008).

[31] R. Sharif, S. Shamaila, F. Shaheen, S. Naseem, J. Y. Chen, M. Khaleeq-ur Rahman, K. Hussain, and X. F. Han, Nanotube wall thickness dependent magnetization reversal properties of NiFe nanotubes, J. Appl. Phys. 113, 024315 (2013).

[32] M. Wyss, A. Mehlin, B. Gross, A. Buchter, A. Farhan, M. Buzzi, A. Kleibert, G. Tütüncüoglu, F. Heimbach, A. Fontcuberta i Morral, D. Grundler, and M. Poggio, Imaging magnetic vortex configurations in ferromagnetic nanotubes, Phys. Rev. B 96, 024423 (2017).

[33] A. Mehlin, B. Gross, M. Wyss, T. Schefer, G. Tütüncüoglu, F. Heimbach, A. Fontcuberta i Morral, D. Grundler, and M. Poggio, Observation of end-vortex nucleation in individual ferromagnetic nanotubes, Phys. Rev. B 97, 134422 (2018).

[34] F.-S. Guo, B. M. Day, Y.-C. Chen, M.-L. Tong, A. Mansikkamäki, and R. A. Layfield, Magnetic hysteresis up to 80 kelvin in a dysprosium metallocene single-molecule magnet, Science 362, 1400 (2018).

[35] J. A. Fernandez-Roldan, D. Chrischon, L. S. Dorneles, O. Chubykalo-Fesenko, M. Vazquez, and C. Bran, A comparative study of magnetic properties of large diameter Co nanowires and nanotubes, Nanomaterials 8, 692 (2018).

[36] P. Landeros, S. Allende, J. Escrig, E. Salcedo, D. Altbir, and E. E. Vogel, Reversal modes in magnetic nanotubes, Appl. Phys. Lett. 90, 102501 (2007).

[37] P. Landeros, O. J. Suarez, A. Cuchillo, and P. Vargas, Equilibrium states and vortex domain wall nucleation in ferromagnetic nanotubes, Phys. Rev. B 79, 024404 (2009).

[38] S. Allende, J. Escrig, D. Altbir, E. Salcedo, and M. Bahiana, Angular dependence of the transverse and vortex modes in magnetic nanotubes, Eur. Phys. J. B 66, 37 (2008).

[39] J. Lee, D. Suess, T. Schrefl, K. H. Oh, and J. Fidler, Magnetic characteristics of ferromagnetic nanotube, J. Magn. Magn. Mater. 310, 2445 (2007).

[40] A. P. Chen, J. Gonzalez, and K. Guslienko, Magnetization reversal modes in short nanotubes with chiral vortex domain walls, Materials, Materials 11, 101 (2018).

[41] Y. Li, T. Wang, and Y. Li, The influence of dipolar interaction on magnetic properties in nanomagnets with different shapes, Phys. Status Solidi B 247, 1237 (2010).

[42] B.-Z. Mi, L.-J. Zhai, and L.-L. Hua, Magnon specific heat and free energy of Heisenberg ferromagnetic single-walled nanotubes: Green's function approach, J. Magn. Magn. Mater. 398, 160 (2016).

[43] O. Canko, F. Taşkın, K. Argin, and A. Erdinç, Hysteresis behavior of Blume-Capel model on a cylindrical Ising nanotube, Solid State Commun. 183, 35 (2014).

[44] J. d'Albuquerque e Castro, D. Altbir, J. C. Retamal, and P. Vargas, Scaling Approach to the Magnetic Phase Diagram of Nanosized Systems, Phys. Rev. Lett. 88, 237202 (2002). 
[45] P. Landeros, J. Escrig, D. Altbir, D. Laroze, J. d'Albuquerque e Castro, and P. Vargas, Scaling relations for magnetic nanoparticles, Phys. Rev. B 71, 094435 (2005).

[46] P. O. Lehtinen, A. S. Foster, Y. Ma, A. V. Krasheninnikov, and R. M. Nieminen, Irradiation-Induced Magnetism in Graphite: A Density Functional Study, Phys. Rev. Lett. 93, 187202 (2004).

[47] E. Konstantinova, Theoretical simulations of magnetic nanotubes using Monte Carlo method, J. Magn. Magn. Mater. 320, 2721 (2008).

[48] M. Donahue and D. Porter, OOMMF User's Guide, Version 1.2a3, NIST technical report, 2002.

[49] J. Lehmann, C. Donnelly, P. M. Derlet, L. J. Heyderman, and M. Fiebig, Poling of an artificial magneto-toroidal crystal, Nat. Nanotechnol. 14, 141 (2019).

[50] W. Scholz, K. Guslienko, V. Novosad, D. Suess, T. Schrefl, R. Chantrell, and J. Fidler, Transition from single-domain to vortex state in soft magnetic cylindrical nanodots, J. Magn. Magn. Mater. 266, 155 (2003).
[51] J. A. Otálora, J. A. López-López, P. Vargas, and P. Landeros, Chirality switching and propagation control of a vortex domain wall in ferromagnetic nanotubes, Appl. Phys. Lett. 100, 072407 (2012).

[52] J. A. Otálora, J. A. López-López, A. S. Núñez, and P. Landeros, Domain wall manipulation in magnetic nanotubes induced by electric current pulses, J. Phys.: Condens. Matter 24, 436007 (2012).

[53] J. A. Otálora, M. Yan, H. Schultheiss, R. Hertel, and A. Kákay, Curvature-Induced Asymmetric Spin-Wave Dispersion, Phys. Rev. Lett. 117, 227203 (2016).

[54] I.-R. Jeon and R. Clerac, Controlled association of singlemolecule magnets (SMMs) into coordination networks: Towards a new generation of magnetic materials, Dalton Trans. 41, 9569 (2012).

[55] S. V. Streltsov, Z. V. Pchelkina, D. I. Khomskii, N. A. Skorikov, A. O. Anokhin, Y. N. Shvachko, M. A. Korotin, V. I. Anisimov, and V. V. Ustinov, Nature of the ferromagnetic ground state in the $\mathrm{Mn}_{4}$ molecular magnet, Phys. Rev. B 89, 014427 (2014). 